\title{
Mineral Rock Classification Using Convolutional Neural Network
}

\author{
Shanmuk Srinivas Amiripalli ${ }^{\text {a }}$, Grandhi Nageshwara Rao a , Jahnavi Behara ${ }^{\text {a }}$ K Sanjay \\ Krishna $^{\mathrm{a}}$, Mathurthi pavan venkat durga ram $^{\mathrm{a}}$ \\ ${ }^{a}$ Dept of CSE, GIT, GITAM University, Visakhapatnam, Andhrapradesh ,India
}

\begin{abstract}
The main aim of the research is to build a model that can effectively predict the type of mineral rocks. Rocks can be predicted by observing it is colour, shape and chemical composition. On-site technicians need to apply different techniques on rock sample in order to predict rock type. Technicians need to apply different techniques on rock samples, so it is a time-consuming process, and sometimes the predictions may be accurate, and sometimes predictions may be false. When predictions are false, it might show a negative impact in several ways for workers and organization as well. We considered an image dataset of rock types, namely Biotite, Bornite, Chrysocolla, Malachite, Muscovite, Pyrite, and Quartz. We applied CNN (Convolutional Neural Network) Algorithm to get a better prediction of different mineral rocks. Nowadays, CNN is mainly used for image classification and image recognition tasks.
\end{abstract}

Keywords. Deep learning, convolutional neural networks $(\mathrm{CNN})$, image preprocessing, convolution layer, pooling layer, flatten layer, fully connected layer

\section{Introduction}

The traditional method for rock classification is manual work with many problems like time-consuming and low accuracy. We can reduce the amount of time required and increase the accuracy by using the domains present in developing technology. Already some scholars or students researched image classification. Deep learning is a sub-domain of Machine learning that is used to know how a human brain works on data by creating patterns for each object in data and producing results based on those patterns. It has the capability of learning unsupervised data (un labelled data) using its hierarchical networks. Deep learning has gained much interest from researchers because it can be used for processing unsupervised data. Convolution Neural Network $(\mathrm{CNN})$ is a class of deep learning which is commonly used for image classification, medical problems (examples detection of cancer, brain tumor, etc.), video recognition (for example identifying students in zoom call for proper attendance, etc.). All these tasks can be completed using $\mathrm{CNN}$ with better accuracy when compared to other algorithms. CNN is one of the best techniques used for extracting features from an image. It can automatically extract features from images with high accuracy,

1 Grandhi Nageshwara Rao, Dept of CSE, GIT, GITAM University, Visakhapatnam, Email: nageshwarao1127@gmail.com 
Whereas its predecessors cannot learn or identify features without any human supervision. It has a wide range of applications such as plant classification basing on images, face recognition, handwritten recognition, analyzing documents. So, we used CNN to create a model that would help users predict the type of mineral rock. This paper focuses on the creation of a rock classifier using CNN's concepts. Our dataset contains various images of seven kinds of rock minerals, namely biotite, bornite, chrysocolla, malachite, muscovite, pyrite and quartz, and all of them are col oured images. Binary classification is a type of classification in which an image is related to only two classes. For example, it can be a "dog(or)cat" prediction. Here, if a user provides an image to the model as input, then the model will identify features and then try to predict the result to which class the image relates. Multi-class classification is a type of classification in which an image can be related to n-number of classes, where 'n' depends on the training dataset, for example, "flower" prediction. Here ' $n$ ' depends on how many types of flowers the user provided for training. If the user-provided only sunflower, jasmine, and rose, then the ' $n$ ' value will be 3 . Value of ' $n$ ' should be greater than two $(n>2)$ then only it would be considered as multi-class classification.

Image classification is a process of recognizing the data to which class does it relates to. We can predict the features in an image by looking at it, whereas computers cannot identify images directly. So, we use different algorithms to develop a model that can identify the class of an image. Here class can be any real-time object. For example, it can be an animal, thing, humans, etc., so we provide a set of data to the Algorithm, which is helpful in the model's training process to identify the class to which the given image relates. Images are divided into a training set and a testing set. The testing set will not be included in the training set, and these images will be new images to the model user build. Binary classification is a type of classification in which an image is related to only two classes. For example, it can be a "dog(or)cat" prediction. Here, if a user provides an image to the model as input, then the model will identify features and then try to predict the result to which class the image relates. Multi-class classification is a type of classification in which an image can be related to n-number of classes, where ' $n$ ' depends on the training dataset, for example, "flower" prediction. Here ' $n$ ' depends on how many types of flowers the user provided for training. If the userprovided only sunflower, jasmine, and rose, then the ' $n$ ' value will be 3 . Value of ' $n$ ' should be greater than $\operatorname{two}(n>2)$ then only it would be considered multi-class classification. The human brain has three main parts called Cerebrum, Cerebellum, Brainstem. When humans try to view anything using their eyes, the information is passed to the region called Visual Cortex, present in Cerebrum. The visual cortex contains many layers, and these layers can also be called filters. Each layer has its job to find edges, identify multiple objects in data, vertical edge detection, horizontal edge detection, detect human faces, detect moving objects, etc. Convolution neural network is well-known image recognition. If we give some binary image, some processing will be done, and it looks for feature orientation and gives output. In CNN, we generally use the 3-dimensional image in which every pixel will have three values, separate channel for red, green end blue colour value. The mathematical function of CNN has two functions ( $f$ and $g$ ) resulting from the third function $\left(f^{*} g\right)$ that identifies how the shape of one is changed by the other. This can be achieved by taking a $3 * 3$ stride or window and placing it on the image, and then it is multiplied with a filter so that we can get weights to the features in images. After completing the first matrix multiplication, we need to move our stride to the next column and perform matrix multiplication again. If 
stride reaches the end of a column, then we move stride to the next row. We continue this process till stride reaches to last of the image.

\section{Literature survey}

In [1], a visual technique that gives insight into the function of intermediate feature layers and the operation of the classifier was introduced to know why the CNN model works so well and how it can be improved. In [2], three different networks Alex Net, GoogLeNet, and ResNet50, were applied on the 3 different datasets ImageNet, CIFAR10, and CIFAR100, better in capabilities and limitations. The analysis stated that GoogLeNet and ResNet50 were able to recognize objects with better precision. In [3] Artificial neural networks and other methods of image classification 2007, Techniques Used is Artificial Neural Networks (ANN), Support Vector Machines (SVM), Fuzzy measures, Genetic Algorithms (G.A.), Fuzzy Support Vector Machines (F.S.V.M.). Relevance is This page demonstrates different accuracy values for traditional classification methods. Here images are pre-processed using Artificial Neural Network (ANN) to compare the accuracy with other machine learning algorithms. The future scope was to Improve the automatic techniques and apply CNN for a higher accuracy result. This paper also fails to consider over fitting between models. In [4], they are Pruning Convolution Neural Networks for Image Instance Retrieval 2010. The technique used is Convolution Neural Network (CNN), node pruning. Relevance is in this paper, and the author tried to optimize the default CNN algorithm by adding pruning techniques to them, which results in the loss of accuracy in the neural network and increases the speed by $3 \%$ for evaluating and classifying datasets over 35000 entries. The future scope is that we can try to reduce the loss by using any other optimized techniques. In [5-6], they improved Optimization of Convolution Neural Networks through parameter fine-tuning 2019.

Techniques used are Convolution Neural Network (CNN), hyperparameter tuning. In this paper, the author compares the original $\mathrm{CNN}$ build with another same network that is optimized by using parameter tuning and feature reduction. The future scope is this is a comparative study not much is to be improved. In [7], A method was proposed to predict lung cancer from C.T. scan images by using Convolutional Neural Networks. The experimental study was carried on a real-time dataset collected from IraqOncology Teaching Hospital/National Centre for Cancer Diseases (IQ-OTH/NCCD). The model achieved 95\% accuracy. In [8], A new methodology was proposed to detect real-time gender classification based on facial images. In the newly proposed methodology, the number of processing layer was reduced. Unlike in conventional $\mathrm{CNN}$, convolution operation was replaced with cross-relation, which reduced computational loads. It was performed in two datasets i.e., SUMS and AT\&T to which the obtained accuracy were $98.75 \%$ and $99.38 \%$, respectively. In [9], Very Deep Convolutional Neural Network-based image classification using small training samples. Techniques used are Convolutional Neural Network (CNN), batch normalization, regularisation. The author uses regularisation and batch normalization on $\mathrm{CNN}$ to fit small datasets with proper and straightforward modifications and do not need to redesign specific small networks. The future scope is we can think of optimizing the overfitting problem by removing some of the nodes in the network to make it slightly weighted. In [10], Optimization of Convolution Neural Network (CNN) parameters for image classification 2017. A technique used is Convolution Neural Network (CNN). 
Relevance is an approach is specified in order to boost up the accuracy of CNN by two steps. One is to add the number of layers, and another is to decrease the window size of the image. The future scope is this is a comparative study not much is to be improved. Optimization can also be achieved by TGO model [11-17].

\section{Proposed Model}

Initially, collect the dataset by downloading images individually or downloading the entire dataset from online websites. The dataset is divided into training and test datasets [18]. Then image pre-processing is applied to the training dataset, which helps the model train well by making all the images into the same size. Users can add extra features like rotating images up to a certain angle and zooming in images up to a specific rate. These features help the model to train well. In training and test datasets, images are placed in their respective sub-folders so that the model would give labels to each sub-folder, and the model returns these labels or numbers as output after the prediction [19].

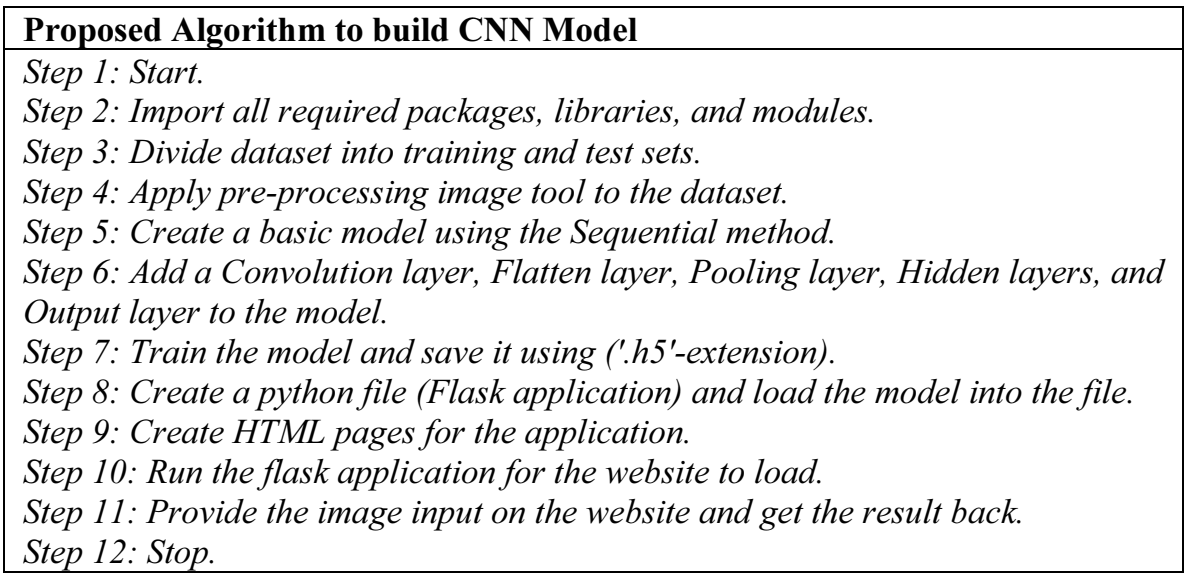

To extract all the features from training images, we need to build a CNN model containing a group of layers. Now add all the layers (specified in the above steps in the same order). With the help of the training dataset, we train the model and save the model. Typically, users need to run entire programs to predict the images, but users can save the model and load that model into a web application. So users do not need to run the program every time for predicting images. Now users can load the model into a web application using respective packages available in python and host their web application. Now users can provide an image as input to application, and then the model will then find features in an image and return the matched class label as output $[20]$. 


\section{Results and Discussion}

We have seven types of mineral rocks in our dataset: biotite, bornite, chrysocolla, malachite, muscovite, pyrite and quartz, and have 951 images (859 images for training and 92 images for testing). We have 951 images for seven classes, so we took $10 \%$ for testing and $90 \%$ for training. We used CNN Algorithm to implement our research because CNN produces better results for image classification. Our model produced an accuracy of $\sim 85 \%$. We applied image pre-processing to images which helped our model to train well [21].

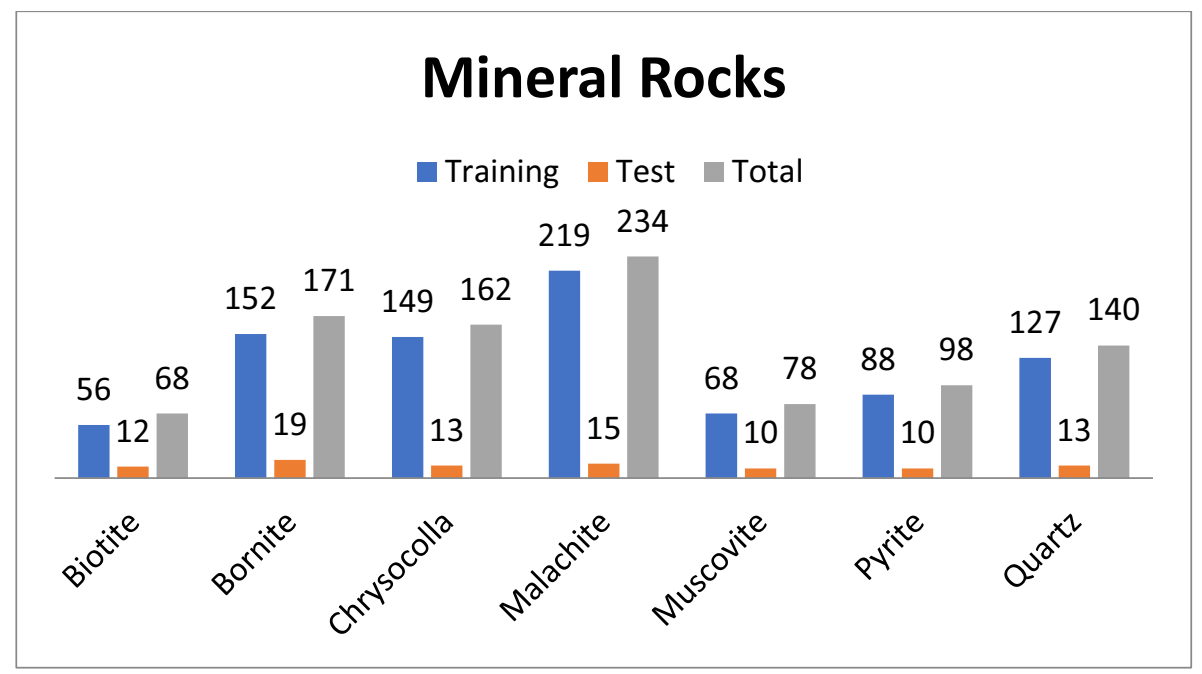

Figure 1. Comparison of mineral rocks dataset with training, test and total.

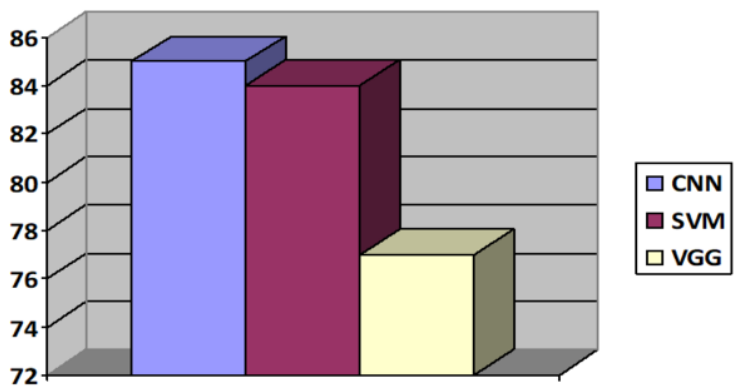

Figure 2. Comparison of accuracy

\section{Conclusion}

This research aims to build a good model that predicts the mineral rock using the images. So, we used CNN for better identification of features in an image. We even applied SVM and V.G.G. algorithms to our dataset. SVM algorithm scores an accuracy of $84 \%$ and the V.G.G. algorithm scores an accuracy of $77 \%$. V.G.G. algorithm 
accuracy can be increased by increasing the number of epochs but the time taken to train the model would be more. We state that our model (CNN algorithm) scored an accuracy of $85 \%$, like the SVM algorithm's accuracy.

\section{References}

[1]. Zeiler, M. D., \& Fergus, R. (2014, September). Visualizing and understanding convolutional networks. In European conference on computer vision (pp. 818-833). Springer, Cham.

[2]. Sharma, N., Jain, V., \& Mishra, A. (2018). An analysis of convolutional neural networks for image classification. Procedia computer science, 132, 377-384.

[3]. Seetha M., Muralikrishna, I. V., Deekshatulu, B. L., Malleswari, B. L., \& Hegde, P. (2008). Artificial neural networks and other methods of image classification. Journal of Theoretical \& Applied Information Technology, 4(11).

[4]. Manek, G., Lin, J., Chandrasekhar, V., Duan, L., Giduthuri, S., Li, X., \&Poggio, T. (2017). Pruning Convolutional Neural Networks for Image Instance Retrieval. arXiv preprint arXiv:1707.05455.

[5]. Becherer, N., Pecarina, J., Nykl, S., \& Hopkinson, K. (2019). Improving optimization of convolutional neural networks through parameter fine-tuning. Neural Computing and Applications, 31(8), 3469-3479.

[6]. Hossain, T., Shishir, F. S., Ashraf, M., Al Nasim, M. A., \& Shah, F. M. (2019, May). Brain Tumor Detection Using Convolutional Neural Network. In 2019 1st International Conference on Advances in Science, Engineering and Robotics Technology (I.C.A.S.E.R.T.) (pp. 1-6). IEEE.

[7]. Potharaju, S. P., \& Sreedevi, M. (2018). A novel subset feature selection framework for increasing the classification performance of SONAR targets. Procedia Computer Science, 125, 902-909.

[8]. Al-Yasriy, H. F., AL-Husieny, M. S., Mohsen, F. Y., Khalil, E. A., \& Hassan, Z. S. (2020, November). Diagnosis of Lung Cancer Based on C.T. Scans Using CNN. In I.O.P. Conference Series: Materials Science and Engineering (Vol. 928, No. 2, p. 022035). I.O.P. Publishing.

[9]. Liew, S. S., Hani, M. K., Radzi, S. A., \&Bakhteri, R. (2016). Gender classification: a convolutional neural network approach. Turkish Journal of Electrical Engineering \& Computer Sciences, 24(3), 1248-1264.

[10]. Liu, S., \& Deng, W. (2015, November). Very deep convolutional neural network-based image classification using small training sample size. In 2015 3rd I.A.P.R. Asian conference on pattern recognition (ACPR) (pp. 730-734). IEEE.

[11]. Sinha, T., Verma, B., \& Haidar, A. (2017). Optimization of convolutional neural network parameters for image classification. In 2017 IEEE Symposium Series on Computational Intelligence (S.S.C.I.) (pp. 1-7). IEEE.

[12]. Potharaju, S. P. (2018). An Unsupervised Approach For Selection of Candidate Feature Set Using Filter Based Techniques. Gazi University Journal of Science, 31(3), 789-799.

[13]. Amiripalli, S. S., Bobba, V., \& Potharaju, S. P.: A novel trimet graph optimization (T.G.O.) topology for wireless networks, (2019) doi:10.1007/978-981-13-0617-4_8.

[14]. Amiripalli, S. S., Kumar, A. K., \& Tulasi, B.: Introduction to T.R.I.M.E.T. along with its properties and scope. In A.I.P. Conference Proceedings (Vol. 1705, No. 1, p. 020032). A.I.P. Publishing L.L.C., (2016).

[15]. Amiripalli, S. S., \& Bobba, V.: Research on network design and analysis of T.G.O. topology. International Journal of Networking and Virtual Organisations, 19(1), 72-86, (2018).

[16]. Amiripalli, S. S., \& Bobba, V.:Trimet graph optimization (T.G.O.) based methodology for scalability and survivability in wireless networks. International Journal of Advanced Trends in Computer Science and Engineering, 8(6), 3454-3460, (2019). doi:10.30534/ijatcse/2019/121862019.

[17]. Amiripalli, S. S., \& Bobba, V. (2019). An Optimal T.G.O. Topology Method for a Scalable and Survivable Network in I.O.T. Communication Technology. Wireless Personal Communications, 107(2), 1019-1040.z

[18]. Amiripalli, S. S., \& Bobba, V.;Impact of trimet graph optimization topology on scalable networks. Journal of Intelligent \& Fuzzy Systems, 36(3), 2431-2442 (2019).

[19]. Amiripalli, S. S, V. Bobba, "A Fibonacci based T.G.O. methodology for survivability in ZigBee topologies". INTERNATIONAL JOURNAL OF SCIENTIFIC \&TECHNOLOGY RESEARCH, 9(2), pp. 878-881. (2020).

[20]. Ramiah Chowdary, P., Challa, Y., Jitendra, M.S.N.V.: "Identification of MITM Attack by Utilizing Artificial Intelligence Mechanism in Cloud Environments" Journal of Physics: Conference Series, 1228 (1),012044, (2019). 
[21]. Thota, J.R., Kothuru, M., Shanmuk Srinivas, A., Jitendra M, S.N.V.: Monitoring diabetes occurrence probability using classification technique with a U.I., International Journal of Scientific and Technology Research, 9 (4), pp. 38-41, (2020).

[22]. Amiripalli, S.S., Venkatarao, R., Jitendra, M.S.N.V., Mycherla, N.M.J.:Detecting emotions of student and assessing the performance by using deep learning International Journal of Advanced Trends in Computer Science and Engineering, 9 (2), pp. 1641-1645, (2020).

[23]. Jitendra, M.S.N.V., Radhika, Y.: A review: Music feature extraction from an audio signal, International Journal of Advanced Trends in Computer Science and Engineering, 9 (2), pp. 973-980, (2020). 